\title{
Niveles de magnesio sérico en pacientes embarazadas con enfermedad hipertensiva manejadas con sulfato de magnesio
}

\author{
Serum magnesium levels in pregnant patients with hypertensive \\ disease managed with magnesium sulfate
}

Héctor Arcos Hernández, * Pablo Mariano González Aldeco, ${ }^{\ddagger}$ Ricardo Pablo Corona ${ }^{\S}$

\section{Resumen}

La eclampsia ocurre del 2 al $3 \%$ en mujeres con preeclampsia con criterios de severidad y que no reciben profilaxis anticonvulsiva, y hasta un $0.6 \%$ en mujeres con preeclampsia sin criterios de severidad. Según la información retrospectiva, se recomienda un rango terapéutico de 4.8 a $8.4 \mathrm{mg} / \mathrm{dL}$. Para el presente trabajo, se realizó un estudio de tipo observacional, retrospectivo y descriptivo, tomando como muestra a las pacientes que han presentado enfermedad hipertensiva del embarazo con indicación para la administración de sulfato de magnesio como profilaxis anticonvulsiva, con dosis impregnación de $4 \mathrm{~g}$ vía intravenosa y de $1 \mathrm{~g} / \mathrm{h}$ de mantenimiento con sulfato de magnesio. Se realizó un estudio retrospectivo en pacientes cuyo diagnóstico fuera enfermedad hipertensiva inducida por el embarazo, que además requirieron manejo con sulfato de magnesio como profilaxis anticonvulsiva, y en quienes se habían tomado niveles séricos de magnesio, analizando los niveles obtenidos. Se encontró que, de las 66 pacientes tratadas con sulfato de magnesio, 56 pacientes (84.4\%) quedaron en niveles infraterapéuticos, 10 pacientes (15.2\%) en niveles terapéuticos, y ninguna paciente con niveles supraterapéuticos.

Palabras clave: Sulfato de magnesio, niveles séricos, niveles terapéuticos, toxicidad, infraterapéuticos, profilaxis anticonvulsiva.
* Médico Residente del Servicio de Ginecología y Obstetricia. Hospital Nuevo Sanatorio Durango.

₹ Jefe de Servicio de Ginecología y Obstetricia del Nuevo Sanatorio Durango.

$\S$ Jefe de Servicio de Ginecología y Obstetricia del Hospital General de Teziutlán, Puebli

Facultad Mexicana de Medicina de la Universidad La Salle. México.

Correspondencia:

Dr. Héctor Arcos Hernández

Correo electrónico: hectorarhe@live.com

Aceptado: 19-11-2019.

www.medigraphic.com/actamedica
Abstract

Eclampsia occurs in 2 to $3 \%$ of women with preeclampsia with non-seizure prophylaxis, and up to $0.6 \%$ of women with preeclampsia without severity criteria. A therapeutic range of 4.8 to $8.4 \mathrm{mg} / \mathrm{dL}$ is recommended according to retrospective information. An observational, retrospective and descriptive study was conducted, taking as a sample those patients who have had hypertensive pregnancy disease with indication for the administration of magnesium sulfate as anticonvulsant prophylaxis, with impregnation dose with $4 \mathrm{~g}$ intravenous and 1 $\mathrm{g} / \mathrm{h}$ maintenance with magnesium sulfate. A retrospective study was conducted in patients diagnosed with pregnancy-induced hypertensive disease who required treatment with magnesium sulfate as anticonvulsant prophylaxis and those who had taken serum magnesium levels, those which were later analyzed. Of the 66 patients treated with magnesium sulfate, 56 patients $(84.4 \%)$ were found to have been in infratherapeutic levels, 10 patients $(15.2 \%)$ at therapeutic levels, and no patients with supratherapeutic levels.

Keywords: Magnesium sulphate, serum levels, therapeutic levels, toxicity, infratherapeutics, anticonvulsant prophylaxis.

\section{INTRODUCCIÓN}

En la antigua Grecia, hacia finales del siglo $V$ e inicios del IV a. C., se hicieron por primera vez las primeras relaciones entre el útero de las mujeres y sus posibles efectos sobre el hígado, el estómago, los pulmones y la cabeza, como posible causal de la enfermedad. Durante la Edad Media y el Renacimiento, debido a la oposición del cristianismo, el avance científico fue mínimo; se cerraron escuelas de medicina en Atenas y Alejandría durante el Imperio bizantino. En el siglo XVIII, Boissier de Sauvages distinguió a la eclampsia de la epilepsia. Para 1849, en el trabajo del Dr. 
William Tyler Smith titulado: Parturition and the Principles and Practice of Obstetrics, se sugirió la posibilidad de congestión cerebral como causa. Si bien durante el siglo XX no se logró encontrar la etiología de la preeclampsia, fue en 1960 donde se encontraron diferencias dramáticas en la fisiología placentaria entre embarazos afectados con preeclampsia y con aquéllos que no. ${ }^{1}$

A nivel mundial, los desórdenes hipertensivos del embarazo constituyen una de las principales causas de mortalidad materna y perinatal; ${ }^{2}$ se estima que globalmente la preeclampsia complica del 2 al $8 \%$ de los embarazos. Aunque la mortalidad materna es mucho menor en países de alto poder adquisitivo en comparación con aquellos países en vías de desarrollo, el 16\% de las muertes maternas puede ser atribuida a desórdenes hipertensivos. ${ }^{3}$ en Estados Unidos, la tasa de preeclampsia aumentó en un $25 \%$ entre 1987 y $2004 ;{ }^{4}$ en comparación con las mujeres embarazadas en 1980, aquellas mujeres en 2003 tenían un riesgo aumentado de 6.7 veces de padecer preeclampsia severa. ${ }^{5}$ Además, se ha reportado que esta complicación es costosa, por ejemplo, en 2012, en Estados Unidos, se estimó un costo de 2.18 billones de dólares 12 meses después del nacimiento (1.03 billones de dólares para las mujeres y 1.15 billones de dólares para los recién nacidos). ${ }^{6}$

La eclampsia ocurre de 2 al 3\% de las mujeres con preeclampsia con criterios de severidad que no reciben profilaxis anticonvulsiva, y hasta un $0.6 \%$ de las mujeres con preeclampsia sin criterios de severidad. ${ }^{7}$ La causa precisa de las convulsiones en la eclampsia aún no está bien comprendida. Existen dos modelos propuestos basados en el rol central de la hipertensión. De acuerdo con el primer modelo, la hipertensión causa un fallo en el sistema de autorregulación de la circulación cerebral, lo que lleva a una hipoperfusión, disfunción endotelial y a un edema vasogénico o citotóxico. En el segundo modelo, la hipertensión causa una activación del sistema autorregulador, llevando a la vasoconstricción de los vasos cerebrales, hipoperfusión, isquemia localizada, disfunción endotelial, y un edema vasogénico o citotóxico. ${ }^{8}$

En la mayoría de las mujeres, se presenta un conjunto de signos y síntomas premonitores horas antes de la convulsión inicial, entre los cuales se encuentran: hipertensión, cefalea, alteraciones visuales, dolor en el cuadrante superior derecho del abdomen o epigastralgia (encontrándose asintomáticas hasta en un $25 \%$ ). 9

La eclampsia generalmente se manifiesta por la presencia de convulsiones tónico-clónicas generalizadas o coma. El patrón de frecuencia cardiaca fetal generalmente se mejora con las intervenciones maternas y fetales. Un patrón no tranquilizante con deceleraciones recurrentes por más de 10 o 15 minutos, a pesar de las intervenciones maternas y fetales, sugiere la posibilidad de un desprendimiento placentario oculto. ${ }^{10}$ Durante la exploración, los hallazgos neurológicos pueden incluir déficit en la memoria, hiperreflexia, déficit visual, estado mental alterado y déficit en los pares craneales. ${ }^{11}$

La eclampsia se presenta pretérmino en un $50 \%$ de los embarazos, y en aproximadamente $20 \%$ de los casos entre las 20 y 30 semanas de gestación. ${ }^{12,13}$ Asimismo, aproximadamente el $90 \%$ de las convulsiones postparto ocurren dentro de una semana del nacimiento. ${ }^{14,15}$

Según un ensayo hecho por Zeeman G y colaboradores, en 2014, los hallazgos por neuroimagen son similares a los vistos en el síndrome de leucoencefalopatía posterior reversible en alrededor de $90 \%$ de las pacientes con eclampsia. ${ }^{16}$ El diagnóstico de eclampsia se hace clínicamente basado en el inicio de convulsiones de nuevo comienzo, tónicoclónicas, focales o multifocales, en ausencia de otra condición (epilepsia, isquemia o infarto cerebrales, hemorragia intracraneal, uso de drogas). ${ }^{17}$ Basado en la información aleatorizada de ensayos clínicos que demuestran que el tratamiento con sulfato de magnesio reduce el riesgo de eclampsia para tratar a todas las mujeres con preeclampsia, se administra como profilaxis anticonvulsiva en el intraparto y postparto. ${ }^{18}$ Es importante hacer énfasis en que la profilaxis anticonvulsiva no previene la progresión de la enfermedad. Aproximadamente, del 10 al $15 \%$ de las mujeres en labor con preeclampsia sin criterios de severidad van a desarrollar signos o síntomas de severidad. ${ }^{19}$

Las grandes organizaciones mundiales constantemente recomiendan el sulfato de magnesio como fármaco de elección para la prevención de eclampsia. ${ }^{20-22}$ En un metaanálisis de estudios aleatorizados de mujeres con preeclampsia, el sulfato de magnesio fue el más efectivo en la prevención de una primera convulsión que en lo casos que fueron tratadas con placebo o sin tratamiento (0.41, $97 \%$ IC 0.29-0.58; seis ensayos, con 11,444 mujeres), fenitoína (RR 0.08, 95\% IC 0.01-0.60; tres ensayos, con 2,291 mujeres), o únicamente un antihipertensivo (nimodipino, RR 0.33, 95\% IC 0.14-0.77; un ensayo, 1,650 mujeres). ${ }^{23}$ Comparado con aquéllas con placebo o sin tratamiento, el sulfato de magnesio resultó ser no estadístico, pero con potencial clínicamente importante en la reducción de muerte materna (RR 0.54, 95\% IC 0.26-1.10).

El mecanismo por el cual el sulfato de magnesio tiene un efecto anticonvulsivo no se ha definido claramente. La hipótesis incluye el aumento del límite en el umbral de la convulsión por su acción en el receptor N-metil Daspartato (NMDA), el cual actúa como un estabilizador de la membrana en el sistema nervioso central secundario a su acción no específica como bloqueador de canal de calcio, así como un reductor de la transmisión de acetilcolina en las terminales neuromotoras. ${ }^{24}$ 
No existe un consenso en el régimen óptimo del sulfato de magnesio acerca de cuándo debe iniciarse o terminarse, así como la ruta de administración. ${ }^{25}$ El régimen de dosificación publicado varía ampliamente, con dosis de impregnación de 4 a 6 g intravenoso durante 20 a 30 minutos, o bien se han mencionado dosis de mantenimiento de 1 a 2 g por horas; ${ }^{26}$ aunque según información retrospectiva, se recomienda un rango terapéutico de 4.8 a $8.4 \mathrm{mg} / \mathrm{dL} .{ }^{27}$ La toxicidad con sulfato de magnesio es poco común en pacientes con función renal normal, ${ }^{28}$ y se correlaciona con la concentración sérica de magnesio. La pérdida de los reflejos osteotendinosos profundos ocurre con 8.5 a $12 \mathrm{mg} / \mathrm{dL}$, parálisis respiratoria con 10 a $13 \mathrm{mg} / \mathrm{dL}$, alteración en la conducción cardiaca > $18 \mathrm{mg} / \mathrm{dL}$ y paro cardiaco con $>25 \mathrm{mg} / \mathrm{dL}^{29}$

\section{Objetivo}

El objetivo primario es identificar la tasa de éxito cuando se alcanzan niveles séricos dentro de rangos terapéuticos en pacientes manejadas con sulfato de magnesio. El esquema a seguir es el descrito por la Guía de práctica clínica para la prevención, diagnóstico y tratamiento de la preeclampsia en segundo y tercer nivel de atención del Instituto Mexicano del Seguro Social, teniendo como objetivos secundarios describir los niveles séricos de magnesio con rango terapéutico con esquema de Zuspan y describir los niveles séricos de magnesio supraterapéuticos.

\section{MATERIAL Y MÉTODOS}

Previa autorización por el Comité de enseñanza en investigación del Hospital General de Teziutlán, Puebla, se realizó un estudio de tipo observacional, retrospectivo y descriptivo. En este estudio se tomó como muestra a las pacientes que han presentado enfermedad hipertensiva del embarazo con indicación para la administración de sulfato de magnesio como profilaxis anticonvulsiva en la misma institución, durante el periodo comprendido de enero de 2017 a mayo de 2019 y a quienes se les midieron niveles séricos de magnesio dentro de las primeras 24 horas, con la finalidad de definir la efectividad alcanzada de los niveles terapéuticos tras la administración de una dosis impregnación con 4 g vía intravenosa y $1 \mathrm{~g} / \mathrm{h}$ de mantenimiento con sulfato de magnesio.

Se eligieron pacientes a quienes se les hubo solicitado niveles séricos de magnesio dentro de las primeras 24 horas posterior al inicio del manejo con sulfato de magnesio y se analizó el porcentaje de pacientes que alcanzan dosis terapéuticas dentro de los niveles establecidos de 4.8 a $8.4 \mathrm{mg} / \mathrm{dL}$.

Los criterios de inclusión fueron:

- Pacientes embarazadas con enfermedad hipertensiva del embarazo.

- Pacientes con diagnóstico de preeclampsia, preeclampsia sobreagregada, preeclampsia con criterios de severidad y síndrome de HELLP.

- Pacientes en quienes se inicia sulfato de magnesio bajo el esquema Zuspan.

- Pacientes a quienes se le tomaron niveles séricos de magnesio dentro de las primeras 24 horas.

Los criterios de exclusión fueron:

- Pacientes diagnosticadas con hipertensión gestacional.

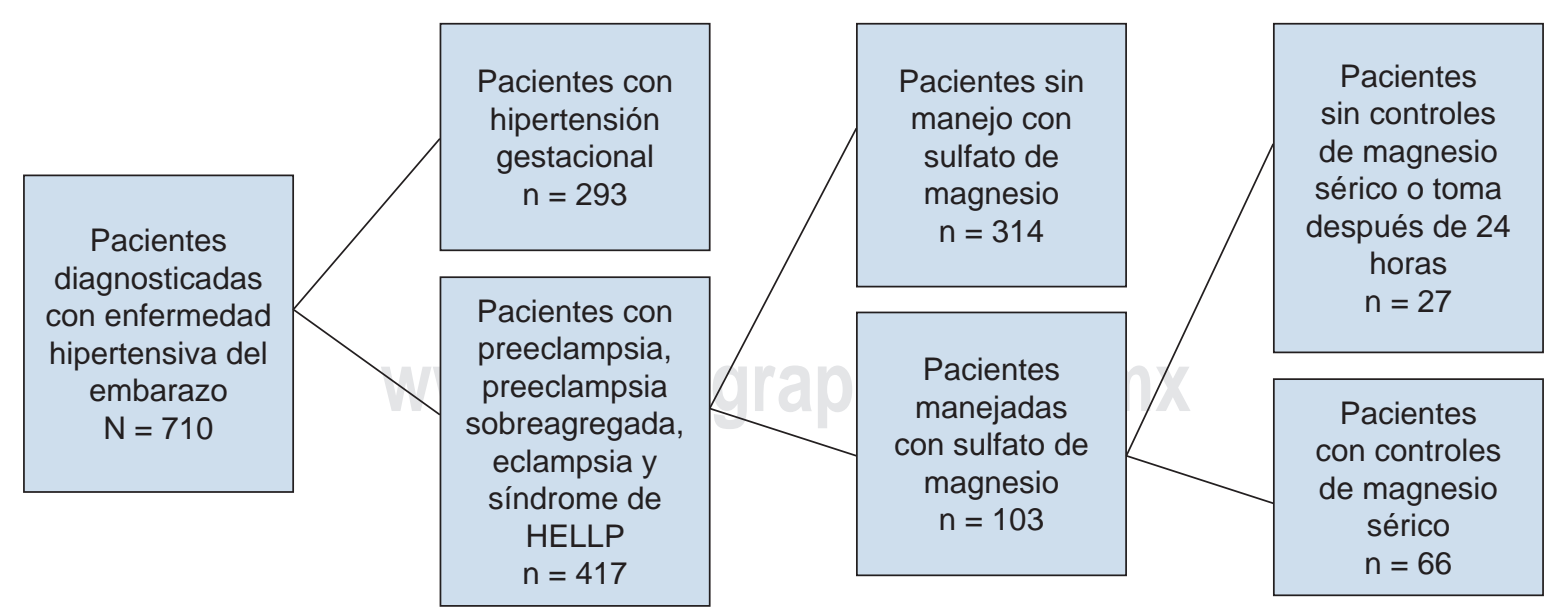

Figura 1: De las 710 pacientes con diagnóstico de enfermedad hipertensiva inducida por el embarazo, se seleccionaron a 66 pacientes que cumplían con los criterios de inclusión. 
- Pacientes en quienes se inicia sulfato de magnesio sin toma de niveles séricos de sulfato de magnesio.

En el análisis estadístico, las variables cualitativas se expresaron en frecuencia y porcentajes; las variables cuantitativas se expresaron en: si tienen libre distribución, con mediana y rango intercuartílico; finalmente, las variables de distribución normal se expresaron en media y desviación estándar tras realizar un análisis de normalidad con la prueba Kolmogórov-Smirnov.

En este estudio, al ser observacional y descriptivo no se tuvo ninguna interacción con las pacientes ni se tuvo influencia en el tratamiento. La búsqueda documental y el llenado del instrumento de recolección respetó la confidencialidad de los datos y se mantuvo la información bajo el resguardo del investigador principal, con fundamento en lo dispuesto por los artículos 13 Inciso A, Fracción I, 14 y 96 de la Ley General de Salud e Inciso A, el artículo 113 al 120 del Reglamento de la Ley General de Salud en Materia de Investigación para la Salud y del artículo 22 Fracción $V$ del Reglamento Interior de la Secretaría de Salud.

De acuerdo con la Ley General de Salud, el estudio se considera de riesgo mínimo por recolectar los datos necesarios a través de procedimientos comunes en exámenes físicos o psicológicos de diagnósticos o tratamientos rutinarios.

\section{RESULTADOS}

Se obtuvo una muestra de 710 pacientes obstétricas que cursaron con diagnóstico de enfermedad hipertensiva del embarazo en el periodo ya estipulado previamente. De ellas, 417 pacientes cursaron con enfermedad hipertensiva del embarazo de tipo preeclampsia, preeclampsia sobreagregada, eclampsia o síndrome de HELLP. Se inició el manejo con sulfato de magnesio en 103 pacientes, de las cuales a 66 pacientes se tomaron controles de forma aleatoria dentro de las primeras 24 horas.

Dentro de las variables generales, la media de IMC encontrada es de $29.42 \pm 4.16$, con una media de talla de $1.52 \pm 0.06 \mathrm{~m}$ y media de peso de $67.89 \pm 9.8 \mathrm{~kg}$.

Se encontró que, de las 66 tratadas con sulfato de magnesio, 56 pacientes $(84.4 \%)$ quedaron en niveles infraterapéuticos, 10 pacientes (15.2\%) en niveles terapéuticos, y ninguna paciente con niveles supraterapéuticos.

Se agrupó a las pacientes por medida de obesidad; la media de niveles séricos de magnesio en pacientes con un IMC normal (10 pacientes o 15.6\%) fue de 3.4 (2.13.77); en pacientes con IMC con sobrepeso (28 pacientes o $43.8 \%$ ) fue de 3.75 (3.3-4.22); en pacientes con IMC con obesidad grado 1 (20 pacientes o 31.3\%) fue de 3.9
(3.12-5.4), y en pacientes con IMC con obesidad grado 2 (6 pacientes o $9.4 \%$ ) fue de 3.55 (3.27-5.1).

Los resultados arrojan que 56 pacientes (84.8\%) obtuvieron niveles infraterapéuticos, mientras que en 10 pacientes (15.2\%) se logró alcanzar niveles terapéuticos.

\section{DISCUSIÓN}

Existe poca bibliografía al respecto que muestre las dosis recomendadas de sulfato de magnesio. Ésta es una de las razones que brinda fortaleza a este estudio; sin embargo, al no ser un estudio prospectivo ni aleatorizado, puede considerarse como una debilidad.

Actualmente, el sulfato de magnesio es un fármaco utilizado de manera empírica, ya que aún no existe un consenso sobre la dosis ideal, tanto de impregnación como de mantenimiento. Según se menciona en el último boletín de práctica de enero 2019 del Colegio Americano de Obstetricia y Ginecología, donde hacen referencia a un estudio de Dayicioglu $\vee$ y su equipo de investigación, quienes, en 2013, relacionaron el índice de masa corporal con la dosis recomendada a administrarse en las pacientes. En este estudio, se concluye que la dosis de mantenimiento recomendada es de $4.5 \mathrm{~g}$ de impregnación y que $1.8 \mathrm{~g}$ produce niveles de magnesio aceptables para la mayoría de las pacientes, pero por debajo del percentil 50 del rango terapéutico recomendado. También se menciona que en pacientes con índice de masa corporal por mayor a 30, esta dosis puede ser insuficiente, recomendándose en esos casos una infusión de 2-2.5 g/h.

Lo que se propone en este estudio es fortalecer la inquietud de definir la dosis ponderal e ir descifrando variables que puedan ejercer efecto en las concentraciones plasmáticas de magnesio en pacientes tratadas con sulfato de magnesio. Consideramos que se requieren más estudios para poder identificar las dosis óptimas de sulfato de magnesio.

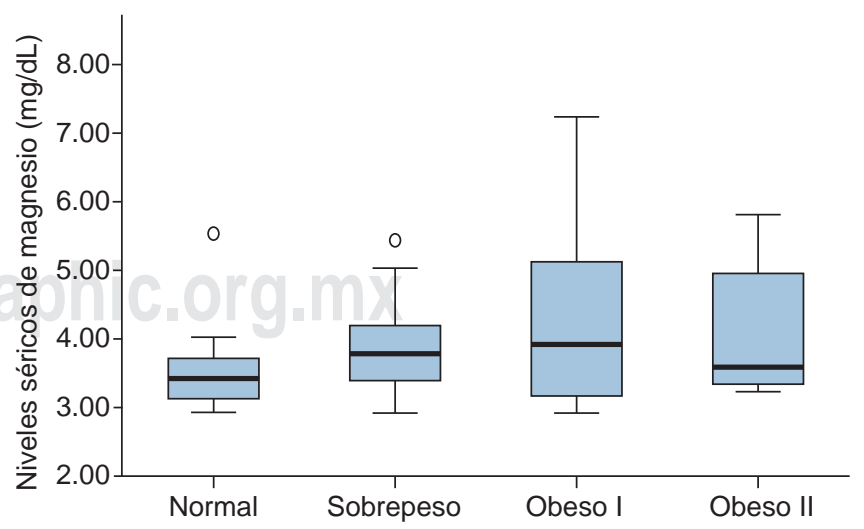

Figura 2: Diagrama de cajas simple de niveles séricos de magnesio (mg/dL por grupo de IMC). 


\section{CONCLUSIÓN}

Las dosis de sulfato de magnesio de acuerdo con la Guía de práctica clínica para la prevención, diagnóstico y tratamiento de la preeclampsia en segundo y tercer nivel de atención se describe como el esquema de Zuspan (4 gramos de sulfato de magnesio intravenoso para 30 minutos seguido de 1 gramo por hora), el cual logra niveles séricos de magnesio en niveles terapéuticos en solamente $15.2 \%$ los casos, siendo una dosis insuficiente para el resto de las pacientes, lo que representa el $84.8 \%$ de los casos.

\section{REFERENCIAS}

1. Bell MJ. A historical overview of preeclampsia-eclampsia. J Obstet Gynecol Neonatal Nurs. 2010; 39 (5): 510-518.

2. Steegers EA, von Dadelszen P, Duvekot JJ, Pijnenborg R. Preeclampsia. Lancet. 2010; 376 (9741): 631-44.

3. Khan K, Wojdyla D, Say L, Gülmezoglu A, Van Look P. WHO analysis of causes of maternal death: a systematic review. Lancet. 2006; 367 (9516): 1066-1074.

4. Wallis AB, Saftlas AF, Hsia J, Atrash HK. Secular trends in the rates of preeclampsia, eclampsia, and gestational hypertension, United States, 1987-2004. Am J Hypertens. 2008; 21 (5): 521-526.

5. Ananth CV, Keyes KM, Wapner RJ. Pre-eclampsia rates in the United States, 1980-2010: age-period-cohort analysis. BMJ. 2013; 347: f6564.

6. Stevens W, Shih T, Incerti D, Ton TGN, Lee HC, Peneva D et al. Short-term costs of preeclampsia to the United States health care system. S Am J Obstet Gynecol. 2017; 217 (3): 237-248.e16.

7. Sibai BM. Magnesium sulfate prophylaxis in preeclampsia: Lessons learned from recent trials. Am J Obstet Gynecol. 2004; 190 (6): 1520-1526.

8. Marra A, Vargas M, Striano P, Del Guercio L, Buonanno P, Servillo G. Posterior reversible encephalopathy syndrome: the endothelial hypotheses. Med Hypotheses. 2014; 82 (5): 619-622.

9. Berhan Y, Berhan A. Should magnesium sulfate be administered to women with mild pre-eclampsia? A systematic review of published reports on eclampsia. J Obstet Gynaecol Res. 2015; 41 (6): 831-842.

10. Sibai BM. Diagnosis, prevention, and management of eclampsia. Obstet Gynecol. 2005; 105 (2): 402-410.

11. Shah AK, Rajamani K, Whitty JE. Eclampsia: a neurological perspective. J Neurol Sci. 2008; 271 (1-2): 158-167.

12. Douglas KA, Redman CW. Eclampsia in the United Kingdom. BMJ. 1994; 309 (6966): 1395-1400.
13. Aagaard-Tillery KM, Belfort MA. Eclampsia: morbidity, mortality, and management. Clin Obstet Gynecol. 2005; 48 (1): 12-23.

14. Dahmus MA, Barton JR, Sibai BM. Cerebral imaging in eclampsia: magnetic resonance imaging versus computed tomography. Am J Obstet Gynecol. 1992; 167 (4 Pt 1): 935-941.

15. Al-Safi Z, Imudia AN, Filetti LC, Hobson DT, Bahado-Singh RO, Awonuga AO. Delayed postpartum preeclampsia and eclampsia: demographics, clinical course, and complications. Obstet Gynecol. 2011; 118 (5): 1102-1107.

16. Zeeman GG, Cunningham FG. Posterior reversible encephalopathy syndrome in 46 of 47 patients with eclampsia. Am J Obstet Gynecol. 2014; 210 (4): 378-379.

17. ACOG Practice Bulletin No. 202 Summary: Gestational hypertension and preeclampsia. Obstet Gynecol. 2019; 133 (1): 211-214.

18. Do women with pre-eclampsia, and their babies, benefit from magnesium sulphate? The magpie trial: a randomised placebocontrolled trial. Obstet Gynecol. 2003; 101 (5): 1018.

19. Livingston JC, Livingston LW, Ramsey R, Mabie BC, Sibai BM. Magnesium sulfate in women with mild preeclampsia: a randomized controlled trial. Obstet Gynecol. 2003; 101 (2): 217-220.

20. Sibai BM. Magnesium sulfate prophylaxis in preeclampsia: Lessons learned from recent trials. Am J Obstet Gynecol. 2004; 190 (6): 1520-1526.

21. Jain V. Letter to the editor: diagnosis, evaluation, and management of the hypertensive disorders of pregnancy: executive summary. J Obstet Gynaecol Can. 2015; 37 (9): 774-775.

22. Roberts JM, Villar J, Arulkumaran S. Preventing and treating eclamptic seizures. BMJ. 2002; 325 (7365): 609-610.

23. Duley L, Gülmezoglu AM, Henderson-Smart DJ, Chou D. Magnesium sulphate and other anticonvulsants for women with pre-eclampsia. Cochrane Database Syst Rev. 2010; (11): CD000025.

24. Chiarello DI, Marín R, Proverbio F, Coronado P, Toledo F, Salsoso R et al. Mechanisms of the effect of magnesium salts in preeclampsia. Placenta. 2018; 69: 134-139.

25. Duley L, Matar HE, Almerie MQ, Hall DR. Alternative magnesium sulphate regimens for women with pre-eclampsia and eclampsia. Cochrane Database Syst Rev. 2010; (8): CD007388.

26. ACOG Practice Bulletin No. 202 Summary: gestational hypertension and preeclampsia. Obstet Gynecol. 2019; 133 (1): 211-214.

27. Sibai BM, Lipshitz J, Anderson GD, Dilts PV Jr. Reassessment of intravenous $\mathrm{MgSO} 4$ therapy in preeclampsia-eclampsia. Obstet Gynecol. 1981; 57 (2): 199-202.

28. Smith JM, Lowe RF, Fullerton J, Currie SM, Harris L, Felker-Kantor E. An integrative review of the side effects related to the use of magnesium sulfate for pre-eclampsia and eclampsia management. BMC Pregnancy Childbirth. 2013; 13: 34

29. Lu JF, Nightingale CH. Magnesium sulfate in eclampsia and pre-eclampsia: pharmacokinetic principles. Clin Pharmacokinet. 2000; 38 (4): 305-314. 